\title{
Prevalence of obesity and associated sociodemographic and lifestyle factors in Morocco
}

\author{
K El Rhazi ${ }^{1, *}, C$ Neijari ${ }^{1}$, A Zidouh ${ }^{2}$, R Bakkali $^{2}, M$ Berraho ${ }^{1}$ and P Barberger Gateau ${ }^{3}$ \\ 'Laboratoire d'Epidémiologie, Recherche Clinique et Santé Communautaire, Faculté de Médecine et de \\ Pharmacie, Km2.2 Route de Sidi Harazem, BP 1893, 30000 Fès, Maroc: ${ }^{2}$ Association Lalla Salma de Lutte \\ contre le Cancer, Rabat, Maroc: ${ }^{3}$ Inserm, Univ Victor Segalen Bordeaux 2, Bordeaux, France
}

Submitted 16 December 2009: Accepted 13 May 2010: First published online 6 July 2010

\begin{abstract}
Objective: In Morocco, the association between obesity/overweight and sociodemographic and lifestyle factors is poorly understood. The present study aimed to investigate this association in a representative sample of the Moroccan population aged 18 years and above.

Design: This is a cross-sectional study using a questionnaire including demographic, socio-economic and physical activity items. Height and weight were measured and BMI was computed. The association between obesity (BMI $\geq 30 \cdot 0 \mathrm{~kg} / \mathrm{m}^{2}$ ) or overweight $\left(25 \cdot 0 \leq \mathrm{BMI}<29 \cdot 9 \mathrm{~kg} / \mathrm{m}^{2}\right)$ and the other variables was analysed using multiple binomial logistic regression, separately in men and women.

Setting: The whole Moroccan territory.

Subjects: A total of 2891 subjects took part in the survey (1430 men and 1461 women). Results: The prevalence of obesity was $20.9 \%$ in women and $6.0 \%$ in men $(P<0 \cdot 0001)$. The prevalence of overweight was $32.9 \%$ in women $v .26 \cdot 8 \%$ in men $(P<0 \cdot 0001)$. In women, the risk of obesity and overweight increased with age, with the highest risk being in individuals aged $45-54$ years $(\mathrm{OR}=3 \cdot 02,95 \% \mathrm{CI} 2 \cdot 06,4 \cdot 44)$ compared to individuals $<35$ years old. Married women were more prone to obesity and overweight $(\mathrm{OR}=2 \cdot 42,95 \% \mathrm{CI} 1 \cdot 50,3 \cdot 91)$ than single women. In men, the risk of obesity and overweight increased with average family income (OR $=2 \cdot 62,95 \% \mathrm{CI}$ $1 \cdot 40,4 \cdot 87$ for family income $\geq 5000 \mathrm{MAD} /$ month compared to $<2000 \mathrm{MAD} / \mathrm{month}$ ) and in married persons $(\mathrm{OR}=3 \cdot 75,95 \% \mathrm{CI} 1 \cdot 78,7 \cdot 81)$ compared to single individuals. Conclusions: These results contribute to target groups in whom prevention programmes could be implemented.
\end{abstract}

Obesity and overweight constitute a major public health problem and their prevalence is increasing at an alarming rate worldwide ${ }^{(1)}$. Obesity is a major risk factor for the development of certain chronic diseases and has been related to some hormone-dependent cancers ${ }^{(2,3)}$. Many environmental factors such as lifestyle and poor diet ${ }^{(4,5)}$, age patterns $^{(6)}$, socio-economic factors ${ }^{(7)}$ and a lack of physical activity $^{(8)}$ play an important role in the onset of obesity ${ }^{(9)}$. Changes in diet and inactivity patterns in transitional societies are fuelling the obesity epidemic ${ }^{(10,11)}$.

Socio-economic status (SES) as a marker for lifestyle behaviours is inversely associated with overweight and obesity in developed societies ${ }^{(12)}$ and positively associated in developing countries ${ }^{(13)}$. However, the relationship between SES and obesity is complex and poorly understood in many countries.

In North African countries, obesity is a growing problem, especially as female fatness is a cultural symbol of beauty, fertility and prosperity ${ }^{(13,14)}$. These countries are undergoing a demographic transition ${ }^{(15)}$ and economic development, which have some implications for lifestyle, diet and related health conditions, especially obesity. However, there are some exceptions, such as Tunisia, where the prevalence of obesity becomes quite similar to that found in European countries, especially in women with higher SES, who prefer a slimmer body such as that in Western societies ${ }^{(16,17)}$. The aetiology of obesity in North Africa is still not well understood and few studies shed any light on its development among women ${ }^{(14)}$.

Morocco is a North African country with Mediterranean climate and a population of 30 million. During the past 50 years, the Moroccan population has been undergoing an epidemiological transition ${ }^{(18)}$. A marked shift was also documented in dietary patterns and lifestyle as part of the nutritional transition. This was associated with an increase in overweight and obesity ${ }^{(19)}$. Many studies have been interested in the prevalence of obesity in the general population and in particular groups ${ }^{(20-23)}$. In the most recent national survey in the year 2000 , overweight and obesity were found to occur among $39 \cdot 3 \%$ and $13 \cdot 2 \%$, 
respectively, of individuals aged 20 years and above ${ }^{(23)}$ but their association with SES remains poorly understood. The objective of the present study was to estimate the prevalence of overweight and obesity in a national representative Sample of Moroccan adults and describe its relationship to SES and the lifestyle of subjects.

\section{Population and methods}

\section{Study design}

The present cross-sectional study was carried out in May 2008 on a national random sample of the Moroccan population aged 18 years and above. Sampling procedures were as follows: sample size was calculated on the basis of $15 \%$ risk factor prevalence, $2 \%$ precision, 95\% CI and a cluster effect of 2; and sample size was estimated at 2448 and rounded to 3000 persons to compensate for people refusing to take part or being absent during the survey. The people to be surveyed were selected at random from 150 communes, in clusters of twenty persons per commune. A cluster was defined as a neighbourhood in an urban area and a locality in a rural area. One cluster was selected at random from each commune included in the survey and one person aged 20 years or above from each household of the cluster was selected at random. The total cluster selection was done proportionally to the distribution of the Moroccan population in urban and rural areas (53 and $47 \%$, respectively $)^{(24)}$. Pregnant women and bed-ridden terminally ill people, as well as the mentally ill, were considered ineligible. A person selected at random was not replaced in the case of absence or refusal to take part. Exclusion criteria included individuals unable to provide the requested information or written consent to participate in the study.

\section{Survey procedure}

The interviewers were first trained to the survey in order to standardize the data collected and the anthropometric measurements. They were teamed up in pairs (one man and one woman) and were supervised by members of the survey planning team. The data were collected in the subjects' respective homes during a personal interview. The questionnaire included questions on demographics (age, sex, place of residence, educational level), SES (declared total monthly income of all the members of the household, profession, etc.) and physical activity, which were assessed by means of a detailed frequency questionnaire, including weekdays and weekends. Usual physical activity in work, method of going to and from work, leisure time and sports practice were considered in the questionnaire.

\section{Variables studied}

\section{Anthropometric measurements}

The following measurements were performed: body weight, height, BMI $\left(\mathrm{kg} / \mathrm{m}^{2}\right)$. Body weight was measured using bathroom scales, with the person wearing light clothes and no shoes. Body weight was expressed in kilograms. The bathroom scales were calibrated before and during the study. Size was measured using a height gauge with the subject standing barefoot. Size was expressed in centimetres.

BMI, corresponding to the person's weight divided by the square of the person's height $\left(\mathrm{kg} / \mathrm{m}^{2}\right)$, was used to define underweight (BMI $<18.5 \mathrm{~kg} / \mathrm{m}^{2}$ ), normal weight $\left(\mathrm{BMI} \geq 18.5\right.$ and $<25 \cdot 0 \mathrm{~kg} / \mathrm{m}^{2}$ ), overweight (BMI $\geq 25 \cdot 0$ and $<30 \cdot 0 \mathrm{~kg} / \mathrm{m}^{2}$ ) and obesity (BMI $\geq 30 \cdot 0 \mathrm{~kg} / \mathrm{m}^{2}$ ) according to $\mathrm{WHO}$ recommendations ${ }^{(1)}$.

\section{Sociodemographic and lifestyle variables}

Among determinants of SES, monthly income was used as a class variable into the following categories: $<2000 \mathrm{MAD}$ corresponding about to the guaranteed minimum wage, 2000-4999 MAD, 5000-9999 MAD and $\geq 10000$ MAD (equivalent currency exchange is: $1 \mathrm{MAD}=0 \cdot 09 €$ ). Given the small number of the two latter classes (5000-9999 and $\geq 10000 \mathrm{MAD} / \mathrm{month}$ ), they were grouped in the unvariate analysis. Profession was categorized into five classes: active, retired, unemployed, housewife and student. We also grouped the highest level of education achieved into the following categories: university, secondary level, primary level, Koranic school or informal education and illiterates. In the multivariate analysis, educational level was grouped into three categories (illiterates, 0 school years; primary level and Koranic school or informal education, $\leq 6$ school years; university and secondary level, $>6$ school years). Ages were grouped into 10-year segments ranging as follows: $<25,25-34,35-44,45-54$, 55-64 and $\geq 65$ years or older. Marital status was also grouped into three classes: married, single, divorced or widowed. Physical activity was defined according to WHO guidelines, which recommended that at least 30 min of regular, moderate or intense physical activity on most days reduces the risk of CVD and diabetes, colon cancer and breast cancer ${ }^{(1)}$. A person was therefore considered as active if he/she performed at least $30 \mathrm{~min}$ of physical activity per day. Housing group was classified according to criteria used previously ${ }^{(25)}$ into five categories: luxurious, modern, new medina, old medina, poor housing or slums and rural housing.

\section{Statistical analysis}

Summary statistics were used to describe the study population. Results were reported as percentage and mean and standard deviation. The association between obesity and the determinant factors considered was researched through univariate analysis and then through multiple binomial logistic regression with being obese as the dependent variable in the first model (model 1) and being obese or overweight as the dependent variable in the second model (model 2). Factors associated with inclusion at $\alpha<10 \%$ in univariate analysis were included 
Table 1 Main demographic and socio-economic characteristics according to gender

\begin{tabular}{|c|c|c|c|c|c|c|}
\hline & \multicolumn{2}{|c|}{ All } & \multicolumn{2}{|c|}{ Men } & \multicolumn{2}{|c|}{ Women } \\
\hline & $n$ & $\%$ & $n$ & $\%$ & $n$ & $\%$ \\
\hline \multicolumn{7}{|l|}{ Age groups (years) } \\
\hline$<25$ & 420 & $14 \cdot 5$ & 176 & $12 \cdot 3$ & 244 & $16 \cdot 7$ \\
\hline $25-34$ & 663 & $22 \cdot 9$ & 293 & $20 \cdot 5$ & 370 & $25 \cdot 3$ \\
\hline $35-44$ & 632 & 21.9 & 270 & $18 \cdot 9$ & 362 & $24 \cdot 8$ \\
\hline $45-54$ & 573 & $19 \cdot 8$ & 295 & $20 \cdot 6$ & 278 & $19 \cdot 0$ \\
\hline $55-64$ & 347 & $12 \cdot 0$ & 216 & $15 \cdot 1$ & 131 & $9 \cdot 0$ \\
\hline$\geq 65$ & 256 & 8.9 & 180 & $12 \cdot 6$ & 76 & $5 \cdot 2$ \\
\hline Total & 2891 & $100 \cdot 0$ & 1430 & $100 \cdot 0$ & 1461 & $100 \cdot 0$ \\
\hline \multicolumn{7}{|l|}{ Marital status } \\
\hline Single & 667 & $23 \cdot 2$ & 368 & $25 \cdot 9$ & 299 & $20 \cdot 6$ \\
\hline Married & 1986 & $69 \cdot 2$ & 1028 & $72 \cdot 2$ & 958 & $66 \cdot 1$ \\
\hline Divorced or widowed & 219 & $7 \cdot \overline{6}$ & 27 & $1 . \overline{9}$ & 192 & $13 \cdot 3$ \\
\hline Total & 2872 & $100 \cdot 0$ & 1423 & $100 \cdot 0$ & 1449 & $100 \cdot 0$ \\
\hline \multicolumn{7}{|l|}{ Educational level } \\
\hline Illiterate & 1250 & $43 \cdot 4$ & 427 & $30 \cdot 1$ & 823 & $56 \cdot 4$ \\
\hline Primary school & 588 & $20 \cdot 4$ & 339 & $23 \cdot 9$ & 249 & $17 \cdot 1$ \\
\hline Secondary & 569 & $19 \cdot 8$ & 342 & $24 \cdot 1$ & 227 & $15 \cdot 5$ \\
\hline University & 219 & $7 \cdot 6$ & 143 & $10 \cdot 1$ & 76 & $5 \cdot 2$ \\
\hline Informal education or Koranic school & 252 & $8 \cdot 8$ & 167 & $11 \cdot 8$ & 85 & $5 \cdot 8$ \\
\hline Total & 2878 & $100 \cdot 0$ & 1418 & - & 1460 & $100 \cdot 0$ \\
\hline \multicolumn{7}{|l|}{ Occupational activity } \\
\hline Active & 1250 & $43 \cdot 6$ & 1053 & $73 \cdot 8$ & 197 & $13 \cdot 7$ \\
\hline Retired & 173 & $6 \cdot 0$ & 160 & $11 \cdot 2$ & 13 & 0.9 \\
\hline Unemployed & 212 & $7 \cdot 4$ & 156 & $10 \cdot \overline{9}$ & 56 & 3.9 \\
\hline Housewife & 1114 & 38.9 & - & - & 1113 & $77 \cdot 3$ \\
\hline Student & 117 & $4 \cdot 1$ & 57 & $4 \cdot 0$ & 60 & $4 \cdot 2$ \\
\hline Total & 2866 & $100 \cdot 0$ & 1426 & $100 \cdot 0$ & 1439 & $100 \cdot 0$ \\
\hline \multicolumn{7}{|l|}{ Average family income (MAD/month) } \\
\hline$<2000$ & 1456 & $52 \cdot 5$ & 819 & $58 \cdot 6$ & 637 & $46 \cdot 3$ \\
\hline 2000-4999 & 714 & $25 \cdot 7$ & 389 & $27 \cdot 8$ & 325 & $23 \cdot 6$ \\
\hline 5000-9999 & 159 & $5 \cdot 7$ & 88 & $6 \cdot 3$ & 71 & $5 \cdot 2$ \\
\hline$\geq 10000$ & 89 & $3 \cdot 2$ & 55 & $3 \cdot 9$ & 34 & $2 \cdot 5$ \\
\hline Don't know & 355 & $12 \cdot 8$ & 47 & $3 \cdot 4$ & 308 & $22 \cdot 4$ \\
\hline Total & 2773 & $100 \cdot 0$ & 1398 & $100 \cdot 0$ & 1375 & $100 \cdot 0$ \\
\hline \multicolumn{7}{|l|}{ Housing } \\
\hline Luxurious or modern & 450 & $15 \cdot 5$ & 234 & $16 \cdot 3$ & 216 & $14 \cdot 8$ \\
\hline New medina & 416 & $14 \cdot 4$ & 201 & $14 \cdot 0$ & 215 & $14 \cdot 7$ \\
\hline Old medina & 546 & $18 \cdot 9$ & 263 & $18 \cdot 4$ & 283 & $19 \cdot 3$ \\
\hline Poor housing or slums & 303 & $10 \cdot 5$ & 145 & $10 \cdot 1$ & 158 & $10 \cdot 8$ \\
\hline Rural housing & 1181 & $40 \cdot 8$ & 590 & $41 \cdot 2$ & 591 & $40 \cdot 4$ \\
\hline Total & 2896 & $100 \cdot 0$ & 1433 & $100 \cdot 0$ & 1463 & $100 \cdot 0$ \\
\hline
\end{tabular}

Sample of the adult Moroccan population, 2008.

in the initial multivariate logistic model. Manual descending multivariate logistic regressions were carried out. Factors associated with inclusion at $\alpha<5 \%$ remained in the final multivariate logistic model. Dummy categorical variables were used in logistic regressions. Univariate and multivariate regression analyses were carried out separately for men and women. Adjusted OR and 95\% CI are presented for both sex groups. All statistical analyses were performed using the Statistical Package for Social Sciences statistical software package version $17 \cdot 0$ (SPSS Inc., Chicago, IL, USA).

\section{Results}

In total, out of the 3000 people selected, 2891 (96.5\%) took part in the survey, including 1430 men (49.5\%) and 1461 women $(50 \cdot 5 \%)$. People who are not included in the survey had refused to participate or were absent $(3.5 \%)$. The sample description according to sex is shown in Table 1.

The overall prevalence of obesity was $13.5 \%$, but was much higher in women than in men $(20.9 \% v .6 \cdot 0 \%$, $P<0 \cdot 0001$; Table 2). This prevalence was significantly higher in urban areas $(14.9 \%)$ than rural areas $(11.7 \%$; $P<0 \cdot 001)$, but the difference was significant only in women. The overall prevalence of overweight was $29 \cdot 9 \%$ (32.9\% in women $v .26 \cdot 8 \%$ in men; $P<0 \cdot 0001)$.

In univariate analyses (Table 2 ), the prevalence of obesity increased with age until 64 years in women, but in men the highest prevalence was observed between 45 and 54 years of age. The prevalence of overweight (not including obesity) increased with age until the age of 64 years in men and until the age of 54 years in women. According to educational level, the prevalence of obesity and overweight decreased from illiterate to university level in women, whereas in men the prevalence of obesity varied little with 
Table 2 BMI distribution by sociodemographic and lifestyle characteristics and gender

\begin{tabular}{|c|c|c|c|c|c|c|c|c|c|c|c|c|}
\hline \multirow[b]{2}{*}{ BMI $\left(\mathrm{kg} / \mathrm{m}^{2}\right)$} & \multicolumn{4}{|c|}{ All } & \multicolumn{4}{|c|}{ Men } & \multicolumn{4}{|c|}{ Women } \\
\hline & $n$ & $<25 \cdot 0$ & $25 \cdot 0-29 \cdot 9$ & $\geq 30 \cdot 0$ & $n$ & $<25 \cdot 0$ & $25 \cdot 0-29 \cdot 9$ & $\geq 30 \cdot 0$ & $n$ & $<25 \cdot 0$ & $25 \cdot 0-29 \cdot 9$ & $\geq 30 \cdot 0$ \\
\hline \multicolumn{13}{|l|}{ Origin area } \\
\hline All & 2881 & $56 \cdot 8$ & $29 \cdot 6$ & $13 \cdot 5$ & 1425 & $67 \cdot 3$ & $26 \cdot 7$ & $6 \cdot 0$ & 1456 & $46 \cdot 6$ & $32 \cdot 5$ & $20 \cdot 9$ \\
\hline Rural & 1201 & $61 \cdot 1$ & $27 \cdot 2$ & $11 \cdot 7$ & 595 & $69 \cdot 1$ & $25 \cdot 2$ & $5 \cdot 7$ & 606 & $53 \cdot 3$ & $29 \cdot 2$ & $17 \cdot 5$ \\
\hline Urban & 1680 & $53 \cdot 8$ & $31 \cdot 4$ & $14 \cdot 9$ & 830 & $66 \cdot 0$ & $27 \cdot 8$ & $6 \cdot 1$ & 850 & $41 \cdot 8$ & $35 \cdot \overline{6}$ & $23 \cdot 4$ \\
\hline$P$ & \multicolumn{4}{|c|}{0.0001} & \multicolumn{4}{|c|}{ NS } & \multicolumn{4}{|c|}{0.0001} \\
\hline \multicolumn{13}{|l|}{ Age groups (years) } \\
\hline All & 2876 & $56 \cdot 8$ & $29 \cdot 7$ & $13 \cdot 5$ & 1422 & $67 \cdot 3$ & $26 \cdot 8$ & $5 \cdot 9$ & 1454 & $46 \cdot 6$ & $32 \cdot 5$ & $21 \cdot 0$ \\
\hline$<25$ & 417 & $82 \cdot 5$ & $14 \cdot 6$ & $2 \cdot 9$ & 175 & $92 \cdot 0$ & $8 \cdot 0$ & - & 242 & $75 \cdot 6$ & $19 \cdot 4$ & $5 \cdot 0$ \\
\hline $25-34$ & 661 & $60 \cdot 4$ & $28 \cdot 3$ & $11 \cdot 3$ & 292 & $70 \cdot 5$ & $24 \cdot 0$ & $5 \cdot 5$ & 369 & $52 \cdot 3$ & $32 \cdot 2$ & $16 \cdot 0$ \\
\hline $35-44$ & 628 & $51 \cdot 3$ & $32 \cdot 8$ & $15 \cdot 9$ & 267 & $67 \cdot 0$ & $27 \cdot 7$ & $5 \cdot 2$ & 361 & $39 \cdot 6$ & $37 \cdot 4$ & $23 \cdot 8$ \\
\hline $45-54$ & 568 & $43 \cdot 0$ & $36 \cdot 4$ & $20 \cdot 6$ & 293 & $56 \cdot 0$ & $34 \cdot 5$ & $9 \cdot 6$ & 275 & $29 \cdot 1$ & $38 \cdot 5$ & $32 \cdot 4$ \\
\hline $55-64$ & 346 & $48 \cdot 8$ & $35 \cdot 8$ & $15 \cdot 3$ & 215 & $58 \cdot 6$ & $36 \cdot 7$ & $4 \cdot 7$ & 131 & $32 \cdot 8$ & $35 \cdot 1$ & $32 \cdot 8$ \\
\hline$\geq 65$ & 256 & 60.9 & $26 \cdot 6$ & $12 \cdot 5$ & 180 & $67 \cdot 2$ & 23.9 & 8.9 & 76 & $46 \cdot 6$ & $32 \cdot 9$ & $21 \cdot 1$ \\
\hline$P$ & \multicolumn{4}{|c|}{0.001} & \multicolumn{4}{|c|}{0.001} & \multicolumn{4}{|c|}{0.0001} \\
\hline \multicolumn{13}{|l|}{ Educational level } \\
\hline All & 2863 & $56 \cdot 7$ & $29 \cdot 8$ & $13 \cdot 5$ & 1410 & $67 \cdot 2$ & $26 \cdot 9$ & $6 \cdot 0$ & 1453 & $46 \cdot 6$ & $32 \cdot 6$ & $20 \cdot 9$ \\
\hline Informal education or Koranic school & 250 & $52 \cdot 0$ & $31 \cdot 2$ & $16 \cdot 8$ & 166 & $58 \cdot 4$ & $30 \cdot 7$ & $10 \cdot 8$ & 84 & $39 \cdot 3$ & $32 \cdot 1$ & $28 \cdot 6$ \\
\hline Illiterate & 1242 & $51 \cdot 0$ & $32 \cdot 0$ & $16 \cdot 9$ & 422 & $66 \cdot 4$ & $28 \cdot 0$ & $5 \cdot 7$ & 820 & $43 \cdot 2$ & $34 \cdot 1$ & $22 \cdot 7$ \\
\hline Primary & 587 & $59 \cdot 5$ & $30 \cdot 0$ & $10 \cdot 6$ & 338 & $68 \cdot 6$ & $26 \cdot 6$ & $4 \cdot 7$ & 249 & $47 \cdot 0$ & $34 \cdot 5$ & $18 \cdot 5$ \\
\hline Secondary & 567 & $66 \cdot 7$ & $23 \cdot 1$ & $10 \cdot 2$ & 341 & $73 \cdot 9$ & $20 \cdot 2$ & $5 \cdot 9$ & 226 & $55 \cdot 8$ & $27 \cdot 4$ & $16 \cdot 8$ \\
\hline University & 217 & $61 \cdot 3$ & $31 \cdot 8$ & $6 \cdot \overline{9}$ & 143 & $60 \cdot 1$ & $35 \cdot \overline{7}$ & $4 \cdot 2$ & 74 & 63.5 & $24 \cdot 3$ & $12 \cdot 2$ \\
\hline$P$ & \multicolumn{4}{|c|}{0.0001} & & & 0.006 & & & & 002 & \\
\hline Marital status & & & & & & & & & & & & \\
\hline All & 2857 & $56 \cdot 7$ & $29 \cdot 7$ & $13 \cdot 6$ & 1415 & $67 \cdot 2$ & $26 \cdot 8$ & $6 \cdot 0$ & 1442 & $46 \cdot 5$ & $32 \cdot 5$ & $21 \cdot 0$ \\
\hline Single & 664 & $76 \cdot 8$ & $18 \cdot 7$ & $4 \cdot 5$ & 367 & $81 \cdot 5$ & $16 \cdot 9$ & $1 \cdot 6$ & 297 & $71 \cdot 0$ & $20 \cdot 9$ & $8 \cdot 1$ \\
\hline Married & 1974 & $51 \cdot 5$ & $32 \cdot 7$ & $15 \cdot 8$ & 1021 & $61 \cdot 9$ & $30 \cdot 6$ & $7 \cdot 5$ & 953 & $40 \cdot 3$ & $35 \cdot 0$ & $24 \cdot 7$ \\
\hline Divorced or widowed & 219 & $43 \cdot 4$ & $35 \cdot 6$ & $21 \cdot 0$ & 27 & $74 \cdot 1$ & $18 \cdot 5$ & $7 \cdot 4$ & 192 & $39 \cdot 1$ & $38 \cdot 0$ & $22 \cdot 9$ \\
\hline & & & 0001 & & & & $0 \cdot 0001$ & & & & 0001 & \\
\hline Occupational activity & & & & & & & & & & & & \\
\hline All & 2850 & $56 \cdot 7$ & $29 \cdot 7$ & $13 \cdot 5$ & 1418 & $67 \cdot 3$ & $26 \cdot 7$ & $6 \cdot 0$ & 1432 & $46 \cdot 3$ & $32 \cdot 7$ & $21 \cdot 0$ \\
\hline Active & 1244 & $62 \cdot 8$ & $28 \cdot 7$ & $8 \cdot 5$ & 1046 & $66 \cdot 5$ & $27 \cdot 5$ & $5 \cdot 9$ & 198 & $42 \cdot 9$ & $34 \cdot 8$ & $22 \cdot 2$ \\
\hline Retired & 172 & $49 \cdot 4$ & $39 \cdot 0$ & $11 \cdot 6$ & 160 & $50 \cdot 0$ & $40 \cdot 6$ & $9 \cdot 4$ & 12 & $41 \cdot 7$ & $16 \cdot 7$ & $41 \cdot 7$ \\
\hline Unemployed & 212 & $75 \cdot 9$ & $17 \cdot 9$ & $6 \cdot 1$ & 156 & $80 \cdot 1$ & $14 \cdot 7$ & $5 \cdot 1$ & 56 & $64 \cdot 3$ & $26 \cdot 8$ & 8.9 \\
\hline Housewife & 1108 & $43 \cdot 9$ & $34 \cdot 1$ & $22 \cdot 0$ & - & - & - & - & 1108 & 43.9 & $34 \cdot 1$ & $22 \cdot 0$ \\
\hline Student & 114 & $91 \cdot 2$ & $6 \cdot 1$ & $2 \cdot 6$ & 56 & $94 \cdot 6$ & $5 \cdot 4$ & - & 58 & $87 \cdot 9$ & $06 \cdot 9$ & $5 \cdot 2$ \\
\hline$P$ & & & 0001 & & & & 0.0001 & & & & 0001 & \\
\hline Average family income (MAD/month) & & & & & & & & & & & & \\
\hline All & 2760 & $57 \cdot 3$ & $29 \cdot 5$ & $13 \cdot 2$ & 1391 & $67 \cdot 4$ & $26 \cdot 5$ & $6 \cdot 0$ & 1369 & $47 \cdot 0$ & $32 \cdot 6$ & $20 \cdot 4$ \\
\hline$<2000$ & 1449 & $60 \cdot 8$ & $27 \cdot 8$ & $11 \cdot 4$ & 814 & $71 \cdot 5$ & $24 \cdot 1$ & $4 \cdot 4$ & 635 & $47 \cdot 1$ & $32 \cdot 6$ & $20 \cdot 3$ \\
\hline $2000-5000$ & 710 & $53 \cdot 4$ & $32 \cdot 0$ & $14 \cdot 6$ & 387 & $62 \cdot 3$ & $29 \cdot 7$ & $8 \cdot 0$ & 323 & $42 \cdot 7$ & $34 \cdot 7$ & $22 \cdot 6$ \\
\hline$\geq 5000$ & 247 & $48 \cdot 6$ & $34 \cdot 4$ & $17 \cdot 0$ & 143 & $57 \cdot 3$ & $31 \cdot 5$ & $11 \cdot 2$ & 104 & $36 \cdot 5$ & $38 \cdot 5$ & $25 \cdot 0$ \\
\hline Don't know & 354 & $57 \cdot 1$ & $28 \cdot 2$ & $14 \cdot 7$ & 47 & $70 \cdot 2$ & $27 \cdot 7$ & $2 \cdot 1$ & 307 & $55 \cdot 0$ & $28 \cdot 3$ & $16 \cdot 6$ \\
\hline$P$ & & & .002 & & & & 0.001 & & & & .02 & \\
\hline Housing & & & & & & & & & & & & \\
\hline All & 2881 & $56 \cdot 8$ & $29 \cdot 6$ & $13 \cdot 5$ & 1425 & $67 \cdot 3$ & $26 \cdot 7$ & $6 \cdot 0$ & 1456 & $46 \cdot 6$ & $32 \cdot 5$ & $20 \cdot 9$ \\
\hline Luxurious or modern & 449 & $50 \cdot 1$ & $37 \cdot 0$ & $12 \cdot 9$ & 234 & $56 \cdot 8$ & $37 \cdot 2$ & $6 \cdot 0$ & 215 & $42 \cdot 8$ & $36 \cdot 7$ & $20 \cdot 5$ \\
\hline New medina & 415 & $52 \cdot 0$ & $28 \cdot 9$ & $19 \cdot 0$ & 201 & $66 \cdot 2$ & $26 \cdot 4$ & $7 \cdot 5$ & 214 & $38 \cdot 8$ & $31 \cdot 3$ & $29 \cdot 9$ \\
\hline Old medina & 543 & $54 \cdot 3$ & $30 \cdot 4$ & $15 \cdot 3$ & 262 & $70 \cdot 2$ & $23 \cdot 7$ & $6 \cdot 1$ & 281 & $39 \cdot 5$ & $36 \cdot 7$ & $23 \cdot 8$ \\
\hline Poor housing or slums & 299 & $60 \cdot 2$ & $28 \cdot 8$ & $11 \cdot 0$ & 143 & $71 \cdot 3$ & $23 \cdot 8$ & 4.9 & 156 & $50 \cdot 0$ & $33 \cdot 3$ & $16 \cdot 7$ \\
\hline Rural housing & 1175 & $61 \cdot 4$ & $27 \cdot 0$ & $11 \cdot 7$ & 585 & $69 \cdot 6$ & $24 \cdot 8$ & $5 \cdot 6$ & 590 & $53 \cdot 2$ & $29 \cdot 2$ & $17 \cdot 6$ \\
\hline$P$ & & & 0001 & & & & 0.02 & & & & 0001 & \\
\hline Physical activity ( $\geq 30 \mathrm{~min} / \mathrm{d}$ ) & & & & & & & & & & & & \\
\hline All & 2620 & $58 \cdot 4$ & $29 \cdot 2$ & $12 \cdot 4$ & 1359 & $67 \cdot 8$ & $26 \cdot 3$ & $5 \cdot 9$ & 1261 & $48 \cdot 2$ & $32 \cdot 4$ & $19 \cdot 4$ \\
\hline Yes & 1606 & $61 \cdot 2$ & $28 \cdot 4$ & $10 \cdot 4$ & 918 & $70 \cdot 0$ & $24 \cdot 8$ & $5 \cdot 1$ & 688 & $49 \cdot 4$ & $33 \cdot 1$ & $17 \cdot 4$ \\
\hline No & 1014 & $53 \cdot \overline{8}$ & $30 \cdot 6$ & $15 \cdot 6$ & 441 & $63 \cdot 0$ & $29 \cdot 5$ & $7 \cdot 5$ & 573 & $46 \cdot 8$ & $31 \cdot 4$ & $21 \cdot 8$ \\
\hline$P$ & & & 0001 & & & & 0.02 & & & & $\cdot 15$ & \\
\hline
\end{tabular}

Sample of adult Moroccan population 2008.

$P$ for global $\chi^{2}$ tests.

educational level. The prevalence of overweight increased up to primary level of schooling in women. In men, overweight was much more frequent in those with a university level of education and in persons attending only informal education or Koranic school. Married women and men had the highest prevalence of obesity. In both genders, single persons had the lowest prevalence of both overweight and obesity.

Within the five occupational groups and according to sex, retired subjects were more often obese or overweight than those with other occupational activities (total prevalence $50 \cdot 0 \%$ and $58.4 \%$ in men and women, respectively), which 
Table 3 Adjusted OR for obesity or overweight in women

\begin{tabular}{|c|c|c|c|c|c|c|}
\hline & \multicolumn{3}{|c|}{ Model 1} & \multicolumn{3}{|c|}{ Model 2} \\
\hline & OR & $95 \% \mathrm{Cl}$ & $P$ & OR & $95 \% \mathrm{Cl}$ & $P$ \\
\hline \multicolumn{7}{|l|}{ Age groups (years) } \\
\hline$<35$ & $1 \cdot 00$ & - & $<0.0001$ & $1 \cdot 00$ & - & $<0.0001$ \\
\hline $35-44$ & 1.93 & $1 \cdot 33,2 \cdot 78$ & & $1 \cdot 80$ & $1 \cdot 35,2 \cdot 40$ & \\
\hline $45-54$ & $3 \cdot 02$ & $2 \cdot 06,4 \cdot 44$ & & $2 \cdot 67$ & $1 \cdot 90,3 \cdot 75$ & \\
\hline$\geq 55$ & $2 \cdot 85$ & $1 \cdot 83,4 \cdot 44$ & & $1 \cdot 94$ & $1 \cdot 32,2 \cdot 86$ & \\
\hline \multicolumn{7}{|l|}{ Marital status } \\
\hline Single & $1 \cdot 00$ & - & 0.0001 & $1 \cdot 00$ & - & 0.0001 \\
\hline Married & $2 \cdot 42$ & $1 \cdot 50,3.91$ & & $2 \cdot 45$ & $1 \cdot 77,3 \cdot 38$ & \\
\hline Divorced or widowed & 1.58 & $0 \cdot 86,2 \cdot 88$ & & $2 \cdot 00$ & $1 \cdot 27,3 \cdot 16$ & \\
\hline \multicolumn{7}{|l|}{ Housing } \\
\hline Rural housing & $1 \cdot 00$ & - & 0.0001 & $1 \cdot 00$ & - & 0.0001 \\
\hline Luxurious or modern & $1 \cdot 16$ & $0 \cdot 77,1 \cdot 75$ & & 1.99 & $1 \cdot 35,2 \cdot 91$ & \\
\hline New medina & $2 \cdot 31$ & $1.58,3.37$ & & $2 \cdot 59$ & $1 \cdot 79,3 \cdot 74$ & \\
\hline Old medina & 1.57 & $1 \cdot 09,2 \cdot 24$ & & $2 \cdot 09$ & $1 \cdot 51,2 \cdot 90$ & \\
\hline Poor housing or slums & 0.99 & $0.62,1.62$ & & $1 \cdot 38$ & $0.94,2.03$ & \\
\hline \multicolumn{7}{|l|}{ Educational level } \\
\hline Illiterate & - & - & & $1 \cdot 00$ & - & $0 \cdot 0120$ \\
\hline Primary or informal education or Koranic school & - & - & & $1 \cdot 04$ & $0 \cdot 77,1.41$ & \\
\hline Secondary or university & - & - & & 0.63 & $0.44,0.90$ & \\
\hline
\end{tabular}

Sample of adult Moroccan population, 2008 ( $n$ 1440).

Model 1: obesity as a dependent variable.

Model 2: obesity or overweight as a dependent variable.

may be explained by their older age: $83.7 \%$ of the retired subjects were 55 years old and above $v .16 \cdot 2 \%$ of the active persons $(P<0 \cdot 0001$; Table 2$)$.

A positive relationship was found between the prevalence of obesity and total family income in both genders; as income of the family increased, the prevalence of obesity increased.

According to housing, the prevalence of obesity and overweight increased with the quality of housing. A higher prevalence was observed among individuals who lived in new or old medina or in luxurious or modern houses than in those who lived in poor housing or in slums. The same trend was observed in the whole population and separately in men and women.

The number of persons living in the same house, as an indirect indicator of SES, was not different between obese (mean 5.9; SD 3.0) and non-obese persons (mean 6.1; $\mathrm{SD} 3 \cdot 0)$. The same results were found separately in men (mean 6.2; SD 3.0) and in women (mean 5.7; SD 2.9) and also when we adjusted for the type of housing or on average family income.

The prevalence of obesity appeared to be lower among subjects who practised at least $30 \mathrm{~min}$ of physical activity per day than in the others $(10.4 \% v .15 \cdot 6 \%, P<0 \cdot 0001)$. The same trend was observed separately in men and in women even if it was not significant in women. But this association disappeared after adjusting for average family income and for the other socio-economic factors.

After correction for possible confounding factors including all demographic and socio-economic factors in multivariate regression models separately in men and women, the retained variables in the final manual descending multivariate logistic regression models differed between men and women. However, in women as in men, physical activity did not remain a significant correlate of obesity or overweight in the multivariate models.

In women, physical activity and occupation were no longer associated with obesity in the final model (Table 3). Age remained strongly associated with obesity with the highest risk in individuals aged 45-54 years compared to those aged $<35$ years. According to marital status, married women remained more prone to obesity than single and divorced or widowed women. However, both married and divorced or widowed women were at higher risk of overweight including obesity. Housing was another independent correlate of obesity with a higher risk in women living in new medina. Similar results were observed in the estimation of adjusted OR for obesity or overweight with, in addition to the previous factors, a significantly decreased risk in women with a higher educational level.

Given the small number of divorced or widowed men ( $n$ 27) this category was grouped with the singles in multivariate analyses (Table 4). Age, education and occupation were no longer associated with obesity in men in the final multivariate model. Married men were more prone to obesity than single, divorced or widowed individuals. Average family income was a strong correlate with increasing risk with higher family income, the highest risk being associated with an income of $\geq 5000$ $\mathrm{MAD} / \mathrm{month}$. When the dependent variable was 'obesity or overweight', age group and occupation were both independently associated with obesity or overweight with the highest risk in retired men. Conversely, risk was significantly decreased in unemployed men. 
Table 4 Adjusted OR for obesity or overweight in men

\begin{tabular}{|c|c|c|c|c|c|c|}
\hline & \multicolumn{3}{|c|}{ Model 1} & \multicolumn{3}{|c|}{ Model 2} \\
\hline & OR & $95 \% \mathrm{Cl}$ & $P$ & OR & $95 \% \mathrm{Cl}$ & $P$ \\
\hline \multicolumn{7}{|l|}{ Age groups (years) } \\
\hline$<35$ & - & - & & $1 \cdot 00$ & - & 0.010 \\
\hline $35-44$ & - & - & & $1 \cdot 24$ & $0 \cdot 83,1 \cdot 85$ & \\
\hline $45-54$ & - & - & & $1 \cdot 91$ & $1 \cdot 28,2 \cdot 85$ & \\
\hline$\geq 55$ & - & - & & $1 \cdot 34$ & $0 \cdot 87,2 \cdot 05$ & \\
\hline Marital status & - & - & & & & \\
\hline Single or divorced or widowed & $1 \cdot 00$ & - & 0.0001 & $1 \cdot 00$ & - & 0.009 \\
\hline Married & $3 \cdot 75$ & $1 \cdot 78,7 \cdot 81$ & & $1 \cdot 66$ & $1 \cdot 13,2 \cdot 43$ & \\
\hline \multicolumn{7}{|c|}{ Average family income (MAD/month) } \\
\hline$<2000$ & $1 \cdot 00$ & - & 0.002 & $1 \cdot 00$ & - & 0.001 \\
\hline $2000-4999$ & $2 \cdot 02$ & $1 \cdot 23,3 \cdot 33$ & & 1.57 & $1 \cdot 20,2 \cdot 06$ & \\
\hline$\geq 5000$ & $2 \cdot 62$ & $1 \cdot 40,4 \cdot 87$ & & $1 \cdot 67$ & $1 \cdot 14,2 \cdot 43$ & \\
\hline \multicolumn{7}{|l|}{ Profession } \\
\hline Active or student & - & - & & 1.00 & - & 0.001 \\
\hline Retired & - & - & & $1 \cdot 67$ & $1 \cdot 12,2 \cdot 50$ & \\
\hline Unemployment & - & - & & 0.53 & $0.32,0.88$ & \\
\hline
\end{tabular}

Sample of adult Moroccan population, 2008 ( $n$ 1325).

Model 1: obesity as a dependent variable.

Model 2: obesity or overweight as a dependent variable.

\section{Discussion}

In the present study, we investigated the relationship between obesity and socio-economic factors within a sample from the Moroccan population aged 18 years and above. The overall prevalence of obesity and overweight was $13.5 \%$ and $29 \cdot 9 \%$, respectively, and higher in women and urban areas compared to men and rural areas. The prevalence of obesity in Morocco has increased from 1.6\% in 1984 to $4 \cdot 3 \%$ in 1998 in men and from 6.4\% in 1984 to $16.0 \%$ in 1998 in women aged 20 years and above. In addition, it increased from $5 \cdot 5 \%$ in 1984 to $12 \cdot 2 \%$ in 1998 in the urban population and from $2 \cdot 6 \%$ in 1984 to $7 \cdot 4 \%$ in 1998 in the rural population ${ }^{(19)}$

Gender difference in the prevalence of obesity was found to be highly significant in our population and there are similar findings in previous studies in Morocco ${ }^{(23)}$ and in other countries ${ }^{(26,27)}$.

Age was strongly associated with obesity in women, as previously reported ${ }^{(28)}$. This association between obesity and age can be explained, in part, by parity and postmenopausal status ${ }^{(29)}$. The reduced risk of obesity in younger women may reflect a possible shift in the burden of obesity in women, from the positive association observed in most studies from sub-Saharan Africa ${ }^{(20,21)}$ to the inverse association reported from developed countries. Therefore, younger women, who are more likely to be educated, adopt lifestyles that are less prone to obesity in response to their exposure to the cultures of more developed countries.

Obesity was inversely associated with educational level in our study, which remained after adjustment for confounding variables in women. This may be due to a healthier lifestyle in individuals who have been educated to a higher level and is in accordance with other recent studies ${ }^{(30)}$.
Several studies have underlined the importance of socioeconomic factors and physical activity ${ }^{(31,32)}$. Our finding showed that family income was strongly and positively associated with obesity, especially in men. In women, a strong association between housing and obesity or overweight was observed. Obesity and overweight were more common among the women living in luxurious or modern housing and in new or old medina. This may be explained by lifestyle factors including level of physical activity. Women living in more comfortable houses and urban areas probably spend less time walking and doing heavy housework than those living in poor housing or in rural areas. Similarly, higher income may provide access to more abundant dietary resources and lifestyle choices. Therefore, wealthier men may be consuming larger portions of food because they can afford to do so. Concerning physical activity, our study showed that it decreased with the average family income $(73 \cdot 1 \%$ in $<2000,67 \cdot 5 \%$ in 2000-4999 and $44.9 \%$ in $\geq 5000 \mathrm{MAD} /$ month; $P<0 \cdot 0001)$. Therefore, the wealthier men walked less often because they owned and used cars frequently. Men with higher income did not do chores that were probably physically demanding because they could afford to employ others. Therefore, they were probably expending less energy. They were also spending greater time at work, which was often sedentary, and hence had less time to participate in leisure-time physical activity. People in the highest socioeconomic classes were more prone to adopting a Western way of life, including more meals taken in restaurants or fast foods, and more time spent watching television or working on computers. However, physical activity was not significantly correlated with obesity or overweight in the multivariate models, perhaps because of a lack of accuracy of its measurement in two classes only. As in other stu$\operatorname{dies}^{(33,34)}$, we evidenced a significant difference in the 
prevalence of obesity with marital status in univariate analysis, with more obesity in married, widowed and divorced individuals compared to single persons. This association remained significant in the multivariate analysis in both genders. This may be explained by access to more abundant food and rich dishes. Furthermore, married, widowed or divorced persons were older than the single persons $(24.7 \%$ and $47.0 \%$ among married and widowed or divorced, respectively, were 55 years old and above $v .1 \cdot 1 \%$ among single persons; $P<0 \cdot 0001$ ). Moreover, it is very likely that the cultural preference for female plumpness coupled with physical and cultural barriers to physical activity makes the difference ${ }^{(20)}$.

Housewives and retired persons were the most frequently obese group in our study compared to those who were employed, unemployed and students. These results are concordant with the results of previous studies ${ }^{(26)}$. But this association disappeared after correction for confounding variables, in particular older age.

The main limitation of the present study is its crosssectional design. We cannot therefore demonstrate causality of the associations with the factors evidenced in the present study. The second limitation was a recall bias, especially for income and education level that might be over- or underestimated because data were collected by direct interview. Furthermore, given the lack of data on number of pregnancies and engagement in sport in the present study, we could not ascertain the impact of these variables on overweight and obesity. In particular, a more precise evaluation of the time spent on various professional, household and recreational activities would have allowed a better estimation of physical activity. However, the strength of this nationwide study was that it was representative of the whole Moroccan population since the prevalence of sociodemographic factors was similar to that reported in the last general census of habitat and population in $2004^{(24)}$. Our findings are in accordance with two previous studies in Morocco, one with a sociological approach of Moroccan women's household roles in relation to obesity ${ }^{(35)}$ and the other one conducted in a specific group of Sahraoui women ${ }^{(36)}$. Another strength was the direct measurement of anthropometric data.

In conclusion, the present study contributes to understanding the relationship between the socio-economic factors and obesity and overweight in the Moroccan population. Higher income and better living conditions associated with higher SES are the main risk factors of overweight and obesity. Recognizing these factors may facilitate planning of preventive programmes for reducing obesity in the targeted groups in this society.

\section{Acknowledgements}

The present study was funded by 'Association Lalla Salma de Lutte contre le Cancer - Maroc'. The authors have no conflict of interest. K.E.R. contributed to the conception and design, acquisition of data, analysis and interpretation of data and was involved in drafting the manuscript; C.N. contributed to the conception and design, interpretation of data, was involved in revising the manuscript critically and gave final approval of the version to be published. A.Z. contributed to the conception and design of data; R.B. contributed to the conception and design of data. M.B. made substantial contributions to conception and design of data; P.B.G. was involved in drafting the manuscript and gave final approval of the version to be published. Special thanks to Dr T. Norat (Department of Epidemiology and Public Health, Imperial College London, UK) for reviewing the manuscript.

\section{References}

1. World Health Organization (2000) Obesity: Preventing and Managing the Global Epidemic. Report of a WHO Consultation on Obesity. WHO Technical Report Series no. 894. Geneva: WHO.

2. Pi-Sunyer FX (1993) Medical hazards of obesity. Ann Intern Med 119, 655-660.

3. Khan LK \& Bowman BA (1999) Obesity: a major global public health problem. Am Rev Nutr 19, 13-17.

4. Chen M, You Y, Zhao W et al. (2002) Influences of diet and nutrition on obesity of pre-school children. Wei Sheng Yan јіи 31, 370-372.

5. Shepard TY, Weil KM, Sharp TA et al. (2001) Occasional physical inactivity combined with a high-fat diet may be important in the development and maintenance of obesity in human subjects. Am J Clin Nutr 73, 703-708.

6. Bartali B, Benvenuti E, Corsi AM et al. (2002) Changes in anthropometric measures in men and women across the life-span: findings from the InCHIANTI study. Soz Praventivmed 47, 336-348.

7. Heitmann BL (1999) Occurrence and development of overweight and obesity among adult Danes aged 30-60 years. Ugeskr Laeger 161, 4380-4384.

8. Lindstrom M, Isacsson SO \& Merlo J (2003) Increasing prevalence of overweight, obesity and physical inactivity: two population based studies 1986 and 1994. Eur J Public Health 13, 306-312.

9. Bouchard C (1996) Genetics of obesity in humans: current issues. In The Origin and Consequences of Obesity, CIBA Foundation Symposium, pp. 108-117 [DJ Chadwick and GC Cardew, editors]. Chichester: Wiley.

10. Popkin BM (2001) The nutrition transition and obesity in the developing world. J Nutr 131, 871S-873S.

11. Popkin BM \& Gordon-Larsen P (2004) The nutrition transition: worldwide obesity dynamics and their determinants. Int J Obes Relat Metab Disord 28, Suppl. 3, S2-S9.

12. McLaren L (2007) Socioeconomic status and obesity. Epidemiol Rev 29, 29-48.

13. Monteiro CA, Moura EC, Conde WL et al. (2004) Socioeconomic status and obesity in adult populations of developing countries: a review. Bull World Health Organ 82, 940-946.

14. Mokhtar N, Elati J, Chabir R et al. (2001) Diet culture and obesity in northern Africa. J Nutr 131, 887S-892S.

15. Popkin BM (1993) Nutritional patterns and transitions. Popul Dev Rev 19, 138-157.

16. Kamoun M, Hajem S, Imen S et al. (2008) Prevalence of obesity and overweight in Tunisia on 2001. Tunis Med 86, 649-652. 
17. Tlili F, Mahjoub A, Lefèvre P et al. (2008) Tunisian women's perceptions of desirable body size and chronic disease risk. Ecol Food Nutr 47, 399-414.

18. Jroundi I (2007) Epidemiological transition in Morocco in the past 50 years. Epidemiology 18, S12.

19. Benjelloun S (2002) Nutrition transition in Morocco. Public Health Nutr 5, 135-140.

20. Rguibi M \& Belahsen R (2007) Prevalence of obesity in Morocco. Obes Rev 8, 11-13.

21. Lahmam A, Baali A, Hilali MK et al. (2008) Obesity, overweight and body-weight perception in a High Atlas Moroccan population. Obes Rev 9, 93-99.

22. Belahsen R, Mziwira M \& Fertat F (2004) Anthropometry of women of childbearing age in Morocco: body composition and prevalence of overweight and obesity. Public Health Nutr 7, 523-530.

23. Tazi MA, Abir-Khalil S, Chaouki N et al. (2003) Prevalence of the main cardiovascular risk factors in Morocco: results of a national survey 2000. J Hypertens 21, 897-903.

24. Haut Commissariat au Plan (2004) Recensement Général de la Population et de l'Habitat 2004. http://www.hca.ma/ pubData/Demographie/RGPH/RGPH_Rapport_National.pdf (accessed June 2010).

25. Direction de la planification et des ressources financières, Division de la planification et des études, Service des études et de l'information sanitaire (2003) Enquête sur la population et la Santé Familiale -2003. Protocole http:// doc.abhatoo.net.ma/doc/IMG/pdf/Protocole-PAPFAM-web.pdf (accessed June 2010).

26. Hajian-Tilaki KO \& Heidari B (2007) Prevalence of obesity, central obesity and the associated factors in urban population aged 20-70 years, in the north of Iran: a population-based study and regression approach. Obes Rev 8, 3-10.
27. Xu F, Yin XM, Zhang M et al. (2004) Family average income and body mass index above the healthy weight range among urban and rural residents in regional Mainland China. Public Health Nutr 8, 47-51.

28. Peixoto MD, Benício MH \& Jardim PC (2007) The relationship between body mass index and lifestyle in a Brazilian adult population: a cross-sectional survey. Cad Saude Publica 23, 2694-2740.

29. Tufano A, Marzo P, Enrini R et al. (2004) Anthropometric, hormonal and biochemical differences in lean and obese women before and after menopause. J Endocrinol Invest 27, 648-653.

30. Hajian-Tilaki KO \& Heidari B (2010) Association of educational level with risk of obesity and abdominal obesity in Iranian adults. J Public Health (Oxf) 32, 202-209.

31. Martinez JA, Kearney JM, Kafatos A et al. (1999) Variables independently associated with self-reported obesity in the European Union. Public Health Nutr 2, 125-133.

32. Aal-Isa AN (1999) Dietary and socio-economic factors associated with obesity among Kuwaiti college men. $\mathrm{Br} \mathrm{J}$ Nutr 82, 369-374.

33. Kahn HS, Williamson DF \& Stevens JA (1991) Race and weight change in US women: the roles of socioeconomic and marital status. Am J Public Health 81, 319-323.

34. Sobal J, Rauschenbach B \& Frongillo EA (2003) Marital status changes and body weight changes: a US longitudinal analysis. Soc Sci Med 56, 1543-1555.

35. Batnitzky A (2008) Obesity and household roles: gender and social class in Morocco. Sociol Health Illn 30, $445-462$

36. Rguibi M \& Belahsen R (2006) Body size preferences and sociocultural influences on attitudes towards obesity among Moroccan Sahraoui women. Body Image 3, 395-400. 